\title{
ATOMIC AND MOLECULAR DATA FOR OBSERVATIONS OF THE INTER- STELLAR MEDIUM WITH THE HUBBLE SPACE TELESCOPE
}

\author{
DONALD C. MORTON \\ Herzberg Institute of Astrophysics \\ National Research Council of Canada \\ 100 Sussex Drive \\ Ottawa, Ontario, Canada K1A 0R6
}

The Goddard High Resolution Spectrograph (GHRS) on the HST, with resolving powers up to 94000 or $3.2 \mathrm{~km} \mathrm{~s}^{-1}$, is giving us a new look at interstellar absorption lines in the range 1090 to $3350 \AA$ A (Savage et al 1991, Cardelli et al 1991, Smith et al 1991). The high signal-to-noise ratios possible with the digicon detectors provide precise line profiles suitable for studying individual velocity components. Morton (1991) has summarized the relevant laboratory data on wavelengths and $f$-values for the atomic absorptions from the ground term likely to be detected by the HST, and Hsu and Hayden Smith (1977) have provided a useful list of candidate molecular electronic transitions.

Comparison of cloud components in different atoms and molecules requires accurate laboratory wavelengths. Their uncertainties are within $\pm 0.002 \AA$ for many of the atomic transitions in the GHRS range, and almost all are known within $\pm 0.02 \AA$. The notable exceptions are the weak intersystem transitions of $\mathrm{Ne} \mathrm{V],} \mathrm{S} \mathrm{IV],} \mathrm{Cl} \mathrm{IV],} \mathrm{Cl} \mathrm{V]} \mathrm{and} \mathrm{Ar}$ V]. The important C IV doublet at 1548.195 and $1550.770 \AA$ has uncertainties of $\pm 0.008 \AA$ corresponding to $\pm 1.6 \mathrm{~km} \mathrm{~s}^{-1}$ (Rottman et al 1990).

Transition probabilities with $1 \mathrm{~s}$ errors within $\pm 0.03 \mathrm{dex}$ and good agreement between theoretical and experimental values are becoming available for some of the resonance lines observable with HST, but many remain to be measured to this accuracy, and some important lines are not yet known within \pm 0.3 dex. The most urgent needs for new measurements are listed below.

$\mathrm{Mg}$ II $\lambda \lambda 1241,1240$ : This doublet has an $f$-value $\sim 10^{2}$ times smaller than its Na I counterpart, and is important for interstellar column densities because the lines at 2804 and $2796 \AA$ invariably are saturated. However no one has measured the transition probability of the weak doublet, and the theoretical results range over a factor 5 because of a strong cancellation of terms. If we assume that the damping wings of $\mathrm{Mg}$ II $\lambda 2796$ give the correct column density for $\xi \mathrm{Per}$, the analysis by Cardelli et al (1991) implies that $\log \lambda \mathrm{f}(1239.925)=-0.98$ rather than -0.48 adopted by Morton (1991).

Si II: This ion has seven resonance lines from 1190 and $2336 \AA$ with a range of five in $\log \lambda f$, and two more lines that were observable with Copernicus. This should be an ideal case for the determination of a curve of growth, but there remain serious inconsistencies between some of the experimental and theoretical $f$-values, and between each of these and the strengths of the interstellar lines. New experiments are needed for all the transitions, and particularly for the weak line at $1808 \AA$.

P II: There are large uncertainties in the $f$-values of all the resonance lines of this ion. Three lines at 1533,1302 , and $1153 \AA$ are observable easily with HST. 
Cr II: The a ${ }^{6} \mathrm{~S}-\mathrm{z}^{6} \mathrm{P}^{0}$ triplet at $2066,2062,2056 \AA$ is observed in the interstellar gas and often is used as an indicator of depletion on to grains. There is a good theoretical $f$-value for the multiplet by Aashamar and Luke (1990), but an experimental check would be worthwhile.

Mn II: The a ${ }^{7} \mathrm{~S}$ - y ${ }^{7} \mathrm{P}^{0}$ triplet at $1201,1199,1197 \AA$ appears in interstellar spectra, but the adopted $f$-values were derived by a curve-of-growth comparison with the strengths of the triplet at 2606, 2594, $2577 \AA$. Independent measurements of the ${ }^{7} \mathrm{P}^{0}$ triplet and the weaker $\mathrm{x}{ }^{7} \mathrm{P}_{0}$ triplet at $1164,1163,1162 \AA$ are very desireable.

Fe II: At least 15 resonance lines with a wide range of $f$-values are observable with HST. However only 4 of these lines have reliable experimental $f$-values. Nussbaumer, Pettini, and Storey (1981) calculated many $f$-values, but comparison with the experimentral data indicates that a correction of about -0.12 dex should be applied to the theoretical $\log \lambda$. Laboratory measurements are needed for some of the weaker short-wavelength lines before the Fe II curve of growth and column density can be obtained reliably. At present we must depend on $f$-values derived from interstellar line strengths.

Co II: This ion has not yet been detected as interstellar absorption, but the strongest lines at $2926,1941,1575$, and $1466 \AA$ are candidates. Measurements would be useful to check the calculations of Kurucz (1989).

Ni II: Several resonance absorption lines have been detected in interstellar clouds and in the spectra of quasi-stellar objects. However only the line at $1752 \AA$ has an experimental transition probability.

$\mathrm{Cu}$ II: The line at $1359 \AA$ occurs in interstellar spectra but the lifetime of the upper level calculated by Theodosiou (1986) is 0.22 dex less than the mean of the measurements by Curtis, Engman, and Martinson (1976) and Kono and Hattori (1982).

\section{References}

Aashamar, K., Luke, T.M. 1990, J. Phys. B23, L733.

Cardelli, J.A., Savage, B.D., Bruhweiler F.C., Smith, A.M., Ebbets, D.C., Sembach K.R., and Sofia, U.J. 1991, ApJ. 377, L57.

Curtis, L.J., Engman, B. and Martinson, I. 1976, Phys. Scripta 13, 109.

Hsu, D.K., and Hayden Smith, W., 1977, Spectroscopy Letters 10, 181.

Kono, A., and Hattori, S. 1982, J. Opt. Soc. Am. 72, 601.

Kurucz, R.L. 1989, computer tapes.

Morton, D.C. 1991 ApJS. 77, 119.

Nussbaumer, H., Pettini, M., and Storey, P.J. 1981, AA. 26, 351.

Rottman, G.J., Hassler, D.M., Jones, M.D., Orrall, F.Q. 1990, ApJ. 358, 693.

Savage, B.D., Cardelli, J.A., Bruhweiler, F.C., Smith, A.M., Ebbets, D.C. and Sembach, K.R. 1991, ApJ. 377, L53.

Smith, A.M., Bruhweiler, F.C., Lambert, D.L., Savage, B.D., Cardelli, J.A., Ebbets, D.C., Lyu, C-H., and Sheffer, Y. 1991 ApJ. 377, L64.

Theodosiou, C.E. 1986, J. Opt. Soc. Am. B 3, 1107. 